\title{
Symbolic-Numerical Algorithms for Solving Parabolic Quantum Well Problem with Hydrogen-Like Impurity
}

\author{
S.I. Vinitsky, O. Chuluunbaatar, V.P. Gerdt, A.A. Gusev, and V.A. Rostovtsev \\ Joint Institute for Nuclear Research, Dubna, Russia \\ vinitsky@theor.jinr.ru
}

\begin{abstract}
For parabolic quantum well problem with hydrogen-like impurity a two-dimensional boundary-value problem is formulated in spherical coordinates at fixed magnetic quantum number. Calculational scheme using modified angular prolate spheroidal functions is presented. Symbolic-numerical algorithms for solving the problem are elaborated. The efficiency of the algorithms and their implementation is demonstrated by solving typical test examples and proving the compatibility conditions for asymptotic solutions of scattering problems in spherical and cylindrical coordinates.
\end{abstract}

Keywords: Symbolic-numerical algorithms, parabolic quantum well, hydrogen-like impurity, modified prolate angular spheroidal functions.

\section{Introduction}

In 1] optical absorption into the ground state of GaAs parabolic quantum well and rectangular quantum well with infinitely high walls in the presence of a hydrogen-like impurity was considered. Calculation of the ground state of these quantum wells was carried out using single-parameter variational functions in the cylindrical coordinate system. The upper bounds of these energies were obtained depending on the shift of the Coulomb potential center. The analysis of more complex quantum mechanical models leads to boundary-value problems in a non-standard domain of the configuration space with complex boundary, solved using finite-element method [2]3, or by means of reducing the problem to ordinary differential equations following Kantorovich method 4, known in physics as the adiabatic approach to quantum mechanical problems with slow and fast variables. In the Kantorovich method, the basis functions depend upon the slow variables as parameters and obey the boundary conditions that account for all specific features of the original problem. This provides the efficiency of the method for solving boundary-value problems in a non-standard domain, e.g, in a sector of a circle with mixed boundary conditions [5], as well as in the presence of singular potential against the background of confining potentials of the oscillator type with respect to some independent variables [6]7. The latter determines the potentialities of using the method to analyze low-dimensional quantum mechanical models of semiconductor nanostructures [8]. 
In this paper we present a scheme for solving the boundary-value problem for a parabolic quantum well in the adiabatic representation and in the spherical coordinates. For efficient application of the Kantorovich method we elaborated the following symbolic-numerical algorithms to compute the appropriate quantities to a prescribed accuracy:

- numerical solution of the parametric self-adjoined Sturm-Liouville problem on a bounded interval of the parameter values and calculation of derivatives with respect to the parameter of the eigenfunctions and of the matrix elements (integrals of the eigenfunctions multiplied by their derivatives with respect to the parameter) that appear as variable coefficients in the system of second-order ordinary differential equations (ODPEVP, implemented in FORTRAN [9]),

- asymptotic forms of the eigenfunctions and of the matrix elements that appear as variable coefficients in asymptotic solutions of the boundary-value problem under consideration and in the asymptotic forms of the system of secondorder ordinary differential equations (MATRA, implemented in MAPLE),

- asymptotic forms of the solutions of the system of second-order ordinary differential equations for small and large values of the radial variable needed for solving the corresponding boundary-value problem with the third-type boundary conditions (ASYMRS, implemented in MAPLE),

- numerical solutions of the boundary-value problem for a system of secondorder ordinary differential equations (KANTBP, implemented in FORTRAN[5]).

The paper is organized as follows. In Section 2, the statement of the boundaryvalue problem is given. In Section 3, the procedure MATRA for analytic calculation of asymptotic form of basis functions and matrix elements at large values of the radial variable is described. In Section 4, the procedure ASYMRS for the calculation of asymptotic forms of fundamental solutions of a system of radial equations at large values of radial variable in the analytic form is presented. In Section 5, a test example of numerical calculation of the ground state energy and wave functions with the help of ODPEVP and KANTBP programs is given. The Conclusion outlines further applications of the above set of symbolic-numerical algorithms and programs.

\section{Problem Statement}

The Schrödinger equation describing the parabolic quantum well problem with shifted hydrogen-like impurity in the reduced atomic units and in the spherical coordinates $(r, \eta=\cos \theta, \phi)$ at a fixed magnetic quantum number $m$ reads as [4]

$$
\left(-\frac{1}{r^{2}} \frac{\partial}{\partial r} r^{2} \frac{\partial}{\partial r}+\frac{1}{r^{2}} A(c, b)-\frac{2 q}{r}\right) \psi_{m}(r, \eta)=2 E \psi_{m}(r, \eta) .
$$

Here $A(c, b) \equiv A^{(0)}(c, b)+c^{2}+f$ is the operator of the modified angular functions, which at $b=f=0$ correspond to the angular prolate spheroidal functions [10]

$$
A^{(0)}(c, b)=-\frac{\partial}{\partial \eta}\left(1-\eta^{2}\right) \frac{\partial}{\partial \eta}+\frac{m^{2}}{1-\eta^{2}}+c^{2}\left(\eta^{2}-1\right)-b \eta,
$$


where $c=\omega r^{2}, b=-2 \omega^{2} z_{c} r^{3}$, and $f=\left(\omega z_{c} r\right)^{2}$ are real parameters depending on the harmonic oscillator frequency $\omega$ and the shift $z_{c}$ of the Coulomb charge $q$ along $z$-axis from the origin of the cylindrical frame $(\rho, z, \phi)$ in $\mathbf{R}^{3}$, i.e., $r=$ $\sqrt{\rho^{2}+\left(z-z_{c}\right)^{2}}$. The wave functions $\psi_{m}(r, \eta, b) \equiv \psi_{m i}(r, \eta, b) \equiv \psi_{m i}\left(r, \eta, z_{c}\right)$ at fixed $m$ obey the following conditions at the boundary of the domain $\Omega_{r, \eta}=$ $\Omega(0 \leq r<\infty,-1 \leq \eta \leq 1):$

$$
\begin{aligned}
& \lim _{\eta \rightarrow \pm 1}\left(1-\eta^{2}\right) \frac{\partial \psi_{m}(r, \eta)}{\partial \eta}=0, \text { for } m=0, \text { and } \psi_{m}(r, \pm 1)=0, \text { for } m \neq 0 \\
& \lim _{r \rightarrow 0} r^{2} \frac{\partial \psi_{m}(r, \eta)}{\partial r}=0 .
\end{aligned}
$$

At large $r=r_{\max } \gg 1$ the discrete-spectrum wave functions obey the Dirichlet boundary condition that follows from the asymptotic behavior of the solution

$$
\lim _{r \rightarrow+\infty} r^{2} \psi_{m}(r, \eta)=0 \quad \rightarrow \quad \psi_{m}\left(r_{\max }, \eta\right)=0,
$$

and also the orthonormality condition

$$
\int_{0}^{r_{\max }} \int_{-1}^{1} \psi_{m i}(r, \eta) \psi_{m j}(r, \eta) r^{2} d r d \eta=\delta_{i j}
$$

The solution of (11)-(3) at fixed $m$ is sought in the form of the Kantorovich expansion with respect to the single-parameter functions $\Phi_{j}(\eta ; r) \equiv \Phi_{m j}(\eta ; r)$ :

$$
\psi_{m i}(r, \eta)=\sum_{j=1}^{j_{\max }} \Phi_{m j}(\eta ; r) \chi_{j i}(r)
$$

Here the functions $\chi_{j i}(r)$ are to be found, while the basis functions $\Phi_{j}(\eta ; r) \in$ $F_{r} \sim L_{2}[-1,1]$ are solutions of the eigenvalue problem:

$$
A(c, b) \Phi_{m j}(\eta ; r)=E_{j}(r) \Phi_{m j}(\eta ; r)
$$

The eigenfunctions $\Phi_{m j}(\eta ; r) \equiv \Phi_{m j}\left(r, \eta, z_{c}\right)$ at fixed $m$ obey the symmetry condition $\Phi_{m j}\left(r, \eta, z_{c}\right)=\exp \left(\imath \pi \nu_{m q}\right) \Phi_{m j}\left(r,-\eta,-z_{c}\right)$, where $\nu_{m q} \equiv \nu_{m q}\left(r, z_{c}\right)$ is the real phase, $q$ is the number of zeros in $\eta \in[-1,1]$, in particular, $\nu_{m q}(r, 0)=q$ at $z_{c}=0, E_{j}\left(r, z_{c}\right)=E_{j}\left(r,-z_{c}\right)$, and the boundary conditions with respect to the angular variable $\eta$ at each fixed value of the parameter $r \in \mathbf{R}_{+}^{1}$

$$
\lim _{\eta \rightarrow \pm 1}\left(1-\eta^{2}\right) \frac{\partial \Phi_{m j}(\eta ; r)}{\partial \eta}=0, \text { for } m=0, \text { and } \Phi_{m j}(r, \pm 1)=0, \text { for } m \neq 0
$$

as well as the orthonormality conditions in the interval $\Omega_{\eta}=[-1,1]$ :

$$
\left\langle\Phi_{m i}(\eta ; r) \mid \Phi_{m j}(\eta ; r)\right\rangle_{\Omega_{\eta}}=\int_{-1}^{1} \Phi_{m i}(\eta ; r) \Phi_{m j}(\eta ; r) d \eta=\delta_{i j} .
$$

Note that the eigenvalues $E_{j}(r)$ of the operator $A(c, b)$ from (5) are related to the eigenvalues $\lambda_{j}(r)$ of the operator $A^{(0)}(c, b)$ from (2) by the equality 
$E_{j}(r)=\lambda_{j}(r)+c^{2}+f$. The projection of Eq. (1), using expansion (4), is reduced to the set of $j_{\max }$ ordinary second-order differential equations with respect to the unknown vector function $\chi^{(i)}(r) \equiv\left(\chi_{1 i}(r), \ldots, \chi_{j_{\max } i}(r)\right)$ :

$$
\left(-\frac{1}{r^{d-1}} \mathbf{I} \frac{d}{d r} r^{d-1} \frac{d}{d r}+\frac{\mathbf{U}(r)}{r^{2}}+\mathbf{Q}(r) \frac{d}{d r}+\frac{1}{r^{d-1}} \frac{d r^{d-1} \mathbf{Q}(r)}{d r}-2 E \mathbf{I}\right) \chi^{(i)}(r)=0 .(8)
$$

Here $d=3$ is the dimension of the above space $\mathbf{R}^{3}, \mathbf{I}, \mathbf{U}(r)$, and $\mathbf{Q}(r)$ are $j_{\max } \times j_{\max }$ matrices whose entries are defined by the following relations:

$$
\begin{aligned}
& U_{i j}(r)=r^{2} H_{i j}(r)+\frac{E_{i}(r)+E_{j}(r)}{2} \delta_{i j}-2 q r \delta_{i j} \quad I_{i j}=\delta_{i j}, \\
& H_{i j}(r)=H_{j i}(r)=\left\langle\frac{\partial \Phi_{i}(\eta ; r)}{\partial r} \mid \frac{\partial \Phi_{j}(\eta ; r)}{\partial r}\right\rangle_{\Omega_{\eta}} \\
& Q_{i j}(r)=-Q_{j i}(r)=-\left\langle\Phi_{i}(\eta ; r) \mid \frac{\partial \Phi_{j}(\eta ; r)}{\partial r}\right\rangle_{\Omega_{\eta}} .
\end{aligned}
$$

The discrete-spectrum solutions obey the asymptotic boundary conditions and the orthonormality condition

$$
\begin{aligned}
& \lim _{r \rightarrow 0} r^{d-1} \frac{d \chi^{(i)}(r)}{d r}=0, \quad \lim _{r \rightarrow \infty} r^{d-1} \chi^{(i)}(r)=0 \quad \rightarrow \quad \chi^{(i)}\left(r_{\max }\right)=0 \\
& \int_{0}^{r_{\max }} r^{d-1}\left(\chi^{(i)}(r)\right)^{T} \chi^{(j)}(r) d r=\delta_{i j}
\end{aligned}
$$

Remark 1. The continuity of the eigenfunction $\Phi_{j}(\eta ; r)$ with respect to the parameter $r$ is very important for calculations of the potential matrix elements (9) and their further applications for solution of boundary problems for a system of coupled differential equations (8) as considered in [5]. Hence we required $\Phi_{j}(\eta ; r)>0$ in the vicinity of the right boundary point $\eta=1[9]$.

Remark 2. The formulation of the boundary-value problem of continuous spectrum for the set of Eqs. (8) using asymptotic expansions of the solutions presented below is given in [5]7.

\section{Symbolic Algorithm for Evaluating the Asymptotic Forms of Matrix Elements}

The procedure MATRA computes the asymptotic forms of solutions of the eigenvalue problem (5) together with the matrix elements (9) as expansions in powers of $r$ and $1 / r$ for small and large values of $r$, respectively. Here we consider the case of large $r$.

In step 1 we go from the coordinate $\eta \in[-1,1]$ to the new coordinate $\hat{z} \in$ $\left[\sqrt{\omega}\left(-r+z_{c}\right), \sqrt{\omega}\left(r+z_{c}\right)\right]$ using the formula $r \eta \equiv z^{\prime}=z-z_{c}=\left(\hat{z}-z_{c} \sqrt{\omega}\right) / \sqrt{\omega}$.

In step 2 we construct the asymptotic expansion defined in the domain $\eta \in\left[-\eta_{1}, \eta_{1}\right]$, where $\eta_{1}=O\left(\left(\omega r^{2}\right)^{-1 / 2+\epsilon}\right), 0<\epsilon<1 / 2$. It means that in 
the evaluation of the corresponding integrals we omit exponentially small terms and change the domain from the finite interval $\left[\sqrt{\omega}\left(-r+z_{c}\right), \sqrt{\omega}\left(r+z_{c}\right)\right]$ to the infinite one $(-\infty,+\infty)$.

In step 3 we find the asymptotic solution $\Phi_{j}^{a s}(\hat{z} ; r)$ and $r^{-2} E_{j}(r)=$ $r^{-2}\left(\lambda_{j}(r)+c^{2}+f\right)=\omega \beta_{j}(r)$ as an expansion with $j=n+1$

$$
\Phi_{j}^{a s}(\hat{z} ; r)=\sqrt[4]{\omega} \sqrt{r} \sum_{k=0}^{k_{\max }} \frac{\Phi_{n}^{(2 k)}(\hat{z})}{r^{2 k}}, E_{j}(r)=\sum_{k=0}^{k_{\max }} \frac{E_{n}^{(2 k)}}{r^{2 k}}, \beta_{j}(r)=\sum_{k=0}^{k_{\max }} \frac{\beta_{n}^{(2 k)}}{r^{2 k}} .
$$

Substituting Eq.(12) into Eq. (5) and equating the coefficients at the same powers of $r$, we arrive at a system of recurrence differential equations for evaluating the coefficients $\Phi_{n}^{(2 k)}(\hat{z})$ and $\beta_{n}^{(2 k)}, k=1, \ldots, k_{\max }$ :

$$
L(n) \Phi_{n}^{(2 k)}=f_{n}^{(2 k)}(\hat{z}), \quad L(n)=-\frac{d^{2}}{d \hat{z}^{2}}-(2 n+1)+\hat{z}^{2},
$$

with the initial data $\beta_{n}^{(0)}=2 n+1$, and $\Phi_{n}^{(0)}(\hat{z})$ is a known solution of the problem

$$
L(n) \Phi_{n}^{(0)}(\hat{z})=0, \quad \int_{-\infty}^{+\infty} \Phi_{n}^{(0)}(\hat{z}) \Phi_{n^{\prime}}^{(0)}(\hat{z}) d \hat{z}=\delta_{n n^{\prime}} .
$$

In Eqs. (13) the right-hand sides $f_{n}^{(2 k)}(\hat{z})$ are defined by the relations

$$
\begin{aligned}
& f_{n}^{(2 k)}(\hat{z})=\frac{\left(\hat{z}-z_{c} \sqrt{\omega}\right)^{2}}{\omega} \frac{d^{2} \Phi_{n}^{(2 k-2)}(\hat{z})}{d \hat{z}^{2}}+\frac{2\left(\hat{z}-z_{c} \sqrt{\omega}\right)}{\omega} \frac{d \Phi_{n}^{(2 k-2)}(\hat{z})}{d \hat{z}} \\
& +\sum_{j=1}^{k}\left(a^{(2 j)}\left(\hat{z}, z_{c}\right)-\beta_{n}^{(2 j)}\right) \Phi^{(2 k-2 j)}(\hat{z})=0,
\end{aligned}
$$

where the coefficients $a^{(2 j)}\left(\hat{z}, z_{c}\right)$ are defined by Taylor expansion at large $\omega r^{2}$

$$
\frac{m^{2}}{1-\eta^{2}}=m^{2}\left(1-\frac{\left(\hat{z}-z_{c} \sqrt{\omega}\right)^{2}}{\omega r^{2}}\right)^{-1}=\sum_{j=0}^{k_{\max }} \frac{a^{(2 j)}\left(\hat{z}, z_{c}\right)}{r^{2 j}} .
$$

Note that the coefficients $a^{(2 j)}\left(\hat{z}, z_{c}\right)$ contain the terms of the order of $\hat{z}^{2 l}$ till $l=j$. The orthogonality and normalization conditions follow from (7) and (12)

$$
I_{j j^{\prime}}^{(2 k)}=\sum_{l=0}^{k} \int_{-\infty}^{\infty} \Phi_{n_{l}}^{(2 l)}(\hat{z}) \Phi_{n_{r}}^{(2 k-2 l)}(\hat{z}) d \hat{z}=\delta_{k 0} \delta_{n_{l} n_{r}}
$$

where $n_{l}=j-1, n_{r}=j^{\prime}-1$.

We find the asymptotic expressions of the matrix elements $H_{j j^{\prime}}(r)$ and $Q_{j j^{\prime}}(r)$ from (9) in the form of expansions

$$
Q_{j j^{\prime}}(r)=\sum_{k=1}^{k_{\max }} \frac{Q_{j j^{\prime}}^{(2 k-1)}}{r^{2 k-1}}, \quad H_{j j^{\prime}}(r)=\sum_{k=1}^{k_{\max }} \frac{H_{j j^{\prime}}^{(2 k)}}{r^{2 k}} .
$$


Table 1. Values of the partial sums (27) for $r^{-2} E_{j}(r)$ depending on $k_{\max }$ for $\omega=3$, $m=0, z_{c}=0.4$, and $r=8$. The last row contains the corresponding numerical values (n.v.) calculated by means of ODPEVP 9 .

\begin{tabular}{lllll}
$j$ & \multicolumn{1}{c}{$r^{-2} E_{1}$} & \multicolumn{1}{c}{$r^{-2} E_{2}$} & \multicolumn{1}{c}{$r^{-2} E_{3}$} & \multicolumn{1}{c}{$r^{-2} E_{4}$} \\
\hline$r^{-0} E_{j}^{(0)}$ & 3 & 9 & 15. & 21. \\
$+r^{-2} E_{j}^{(2)}$ & 2.9845312 & 8.9614062 & 14.922656 & 20.868281 \\
$+r^{-4} E_{j}^{(4)}$ & 2.9844843 & 8.9611764 & 14.922034 & 20.866998 \\
$+r^{-6} E_{j}^{(6)}$ & 2.9844838 & 8.9611729 & 14.922022 & 20.866966 \\
$+r^{-8} E_{j}^{(8)}$ & 2.9844838 & 8.9611729 & 14.922022 & 20.866965 \\
$+r^{-10} E_{j}^{(10)}$ & 2.9844838 & 8.9611729 & 14.922022 & 20.866965 \\
\hline (n.v.) & 2.9844838 & 8.9611729 & 14.922022 & 20.866965 \\
\hline
\end{tabular}

Here the coefficients $Q_{j j^{\prime}}^{(2 k+1)}$ and $H_{j j^{\prime}}^{(2 k+2)}$ are defined by the relations

$$
\begin{aligned}
& Q_{j j^{\prime}}^{(2 k+1)}=-\sum_{l=0}^{k} \int_{-\infty}^{+\infty} \Phi_{n_{l}}^{(2 l)}(\hat{z}) \hat{Q} \Phi_{n_{r}}^{(2 k-2 l)}(\hat{z}) d \hat{z} \\
& H_{j j^{\prime}}^{(2 k+2)}=\sum_{l=0}^{k} \int_{-\infty}^{+\infty} \hat{Q} \Phi_{n_{l}}^{(2 l)}(\hat{z}) \hat{Q} \Phi_{n_{r}}^{(2 k-2 l)}(\hat{z}) d \hat{z} \\
& \hat{Q} \Phi_{n_{l}}^{(2 l)}(\hat{z})=\left(\frac{1}{2}-2 l\right) \Phi_{n_{l}}^{(2 l)}(\hat{z})+\left(\hat{z}-z_{c} \sqrt{\omega}\right) \frac{d \Phi_{n_{l}}^{(2 l)}(\hat{z})}{d \hat{z}}
\end{aligned}
$$

In step 4 we construct $\Phi_{n}^{(2 k)}(\hat{z})$ as the expansion with unknown coefficients $b_{n ; s}^{(2 k)}$

$$
\Phi_{n}^{(2 k)}(\hat{z})=\sum_{s=-M(k)}^{M(k)} b_{n ; s}^{(2 k)} \Phi_{n+s}^{(0)}(\hat{z}) .
$$

Here the basis functions $\Phi_{v}^{(0)}(\hat{z})$ are solutions of (14) expressed in terms of the Hermite polynomials 10 .

$$
\Phi_{v}^{(0)}(\hat{z})=\frac{H_{v}(\hat{z}) \exp \left(-\hat{z}^{2} / 2\right)}{\sqrt[4]{\pi} \sqrt{2^{v}} \sqrt{v !}} .
$$

Using the known recurrence relation for Hermite polynomials $H_{v}(\hat{z})$

$$
\hat{z} H_{v}(\hat{z})=\frac{H_{v+1}(\hat{z})}{2}+v H_{v-1}(\hat{z}), \quad \frac{d H_{v}(\hat{z})}{d \hat{z}}=2 v H_{v-1}(\hat{z})
$$

we obtain the recurrence relations for the basis functions $\Phi_{v}^{(0)}(\hat{z})$ :

$$
\begin{aligned}
& \hat{z} \Phi_{v}^{(0)}(\hat{z})=+\frac{\sqrt{v+1}}{\sqrt{2}} \Phi_{v+1}^{(0)}(\hat{z})+\frac{\sqrt{v}}{\sqrt{2}} \Phi_{v-1}^{(0)}(\hat{z}), \\
& \frac{d \Phi_{v}^{(0)}(\hat{z})}{d \hat{z}}=-\frac{\sqrt{v+1}}{\sqrt{2}} \Phi_{v+1}^{(0)}(\hat{z})+\frac{\sqrt{v}}{\sqrt{2}} \Phi_{v-1}^{(0)}(\hat{z}),
\end{aligned}
$$


Table 2. The same as in Table 1 but for $Q_{i j}(r)$ at $i \neq j$

\begin{tabular}{lcccccc}
$i, j$ & $Q_{12}, 10^{-2}$ & $Q_{23}, 10^{-2}$ & $Q_{34}, 10^{-1}$ & $Q_{13}, 10^{-2}$ & $Q_{24}, 10^{-1}$ & $Q_{14}, 10^{-4}$ \\
\hline$r^{-1} Q_{i j}^{(1)}$ & 6.1237243 & 8.6602540 & 1.0606601 & -8.8388347 & -1.5309310 & 0 \\
$+r^{-3} Q_{i j}^{(3)}$ & 6.2072876 & 8.8911941 & 1.1027551 & -8.8450495 & -1.5340009 & -5.5338541 \\
$+r^{-5} Q_{i j}^{(5)}$ & 6.2085282 & 8.8962122 & 1.1040117 & -8.8447852 & -1.5339440 & -5.6676737 \\
$+r^{-7} Q_{i j}^{(7)}$ & 6.2085518 & 8.8963403 & 1.1040530 & -8.8447748 & -1.5339400 & -5.6710585 \\
$+r^{-9} Q_{i j}^{(9)}$ & 6.2085523 & 8.8963441 & 1.1040545 & -8.8447745 & -1.5339398 & -5.6711548 \\
\hline (n.v.) & 6.2085523 & 8.8963442 & 1.1040546 & -8.8447745 & -1.5339398 & -5.6711580 \\
\hline
\end{tabular}

$$
\begin{aligned}
& \hat{z} \frac{d \Phi_{v}^{(0)}(\hat{z})}{d \hat{z}}=-\frac{1}{2} \Phi_{v}^{(0)}(\hat{z})-\frac{\sqrt{v+1} \sqrt{v+2}}{2} \Phi_{v+2}^{(0)}(\hat{z})+\frac{\sqrt{v-1} \sqrt{v}}{2} \Phi_{v-2}^{(0)}(\hat{z}), \\
& L(n) \Phi_{n+s}^{(0)}(\hat{z}) \equiv\left(-\frac{d^{2}}{d \hat{z}^{2}}-(2 n+1)+\hat{z}^{2}\right) \Phi_{n+s}^{(0)}(\hat{z})=2 s \Phi_{n+s}^{(0)}(\hat{z}) .
\end{aligned}
$$

From (13), (15), and (21) we obtain the needed value of $M(k)=2 k+1$ in the expansion (19) to provide the calculation of nonzero terms only.

Substituting Eq. (19) into Eq. (13), using Eq. (21) and equating coefficients at the same powers of $r$, we arrive at a set of recurrence relations for evaluating the coefficients $\beta_{n}^{(2 k)}$ and $b_{n ; s}^{(2 k)}$

$$
\begin{aligned}
& 2 s b_{n ; s}^{(2 k)}=f_{n ; s}^{(2 k)} \\
& f_{n ; s}^{(2 k)}=-\sum_{t=-4}^{4} h_{n ; s-t, t} b_{n ; s-t}^{(2 k-2)}-\sum_{j=1}^{k} \sum_{t=-2 j}^{2 j} a_{n ; s-t, t}^{(2 j)} b_{n ; s-t}^{(2 k-2 j)}+\sum_{j=1}^{k} \beta_{n}^{(2 j)} b_{n ; s}^{(2 k-2 j)} \\
& I_{j j^{\prime}}^{(2 k)}=\sum_{l=0}^{k} \sum_{s=-2 k-1}^{2 k+1} b_{n_{l} ; s}^{(2 l)} b_{n_{r} ; s+n_{l}-n_{r}}^{(2 k-2 l)}=\delta_{k 0} \delta_{n_{l} n_{r}}
\end{aligned}
$$

with the initial data $\beta_{n}^{(0)}=2 n+1$ and $b_{n ; s}^{(0)}=\delta_{s 0}$. The coefficients $h_{n ; s, t}$ and $a_{n ; s, t}^{(2 j)}$ in the relations (22) are calculated using (15), (21) from the relations

$$
\begin{aligned}
& \frac{\left(\hat{z}-z_{c} \sqrt{\omega}\right)^{2}}{\omega} \frac{d^{2} \Phi_{n+s}^{(0)}(\hat{z})}{d \hat{z}^{2}}+\frac{2\left(\hat{z}-z_{c} \sqrt{\omega}\right)}{\omega} \frac{d \Phi_{n+s}^{(0)}(\hat{z})}{d \hat{z}}=\sum_{t=-4}^{4} h_{n ; s, t} \Phi_{n+s+t}^{(0)}(\hat{z}) \\
& a^{(2 j)}\left(\hat{z}, z_{c}\right) \Phi_{n+s}^{(0)}(\hat{z})=\sum_{t=-2 j}^{2 j} a_{n ; s, t}^{(2 j)} \Phi_{n+s+t}^{(0)}(\hat{z}) .
\end{aligned}
$$

The corresponding coefficients $Q_{j j^{\prime}}^{(2 k+1)}$ and $H_{j j^{\prime}}^{(2 k+2)}$ from Eq. (18) have the following explicit form:

$$
Q_{j j^{\prime}}^{(2 k+1)}=-\sum_{l=0}^{k} \sum_{s=-2 k-1}^{2 k+1} b_{n_{l} ; s}^{(2 l)}\left((-2 k+2 l) b_{n_{r} ; s+n_{l}-n_{r}}^{(2 k-2 l)}\right.
$$


Table 3. The same as in Table 1 but for $H_{i j}(r)$ at $i \neq j$

\begin{tabular}{lrrrrrr}
$i, j$ & $H_{12}, 10^{-3}$ & $H_{23}, 10^{-2}$ & $H_{34}, 10^{-2}$ & $H_{13}, 10^{-3}$ & $H_{24}, 10^{-2}$ & $H_{14}, 10^{-2}$ \\
\hline$r^{-2} H_{i j}^{(2)}-7.6546554$ & -2.1650635 & -3.9774756 & -5.3033008 & -0.9185586 & 1.8750000 \\
$+r{ }^{-4} H_{i j}^{(4)}$ & -7.7794422 & -2.2165683 & -4.1102192 & -5.6189439 & -1.0089114 & 1.9299804 \\
$+r{ }^{-6} H_{i j}^{(6)}$ & -7.7817944 & -2.2178064 & -4.1142837 & -5.6289351 & -1.0129576 & 1.9313221 \\
$+r{ }^{-8} H_{i j}^{(8)}-7.7818496$ & -2.2178410 & -4.1144203 & -5.6292488 & -1.0131247 & 1.9313584 \\
$+r^{-10} H_{i j}^{(10)}-7.7818511$ & -2.2178422 & -4.1144255 & -5.6292592 & -1.0131316 & 1.9313595 \\
\hline (n.v.) & -7.7818512 & -2.2178422 & -4.1144257 & -5.6292596 & -1.0131319 & 1.9313596 \\
\hline
\end{tabular}

$$
\begin{aligned}
& +\frac{z_{c} \sqrt{\omega} \sqrt{n_{l}+s}}{\sqrt{2}} b_{n_{r} ; s+n_{l}-n_{r}-1}^{(2 k-2 l)}-\frac{z_{c} \sqrt{\omega} \sqrt{n_{l}+s+1}}{\sqrt{2}} b_{n_{r} ; s+n_{l}-n_{r}+1}^{(2 k-2 l)} \\
& \left.-\frac{\sqrt{n_{l}+s-1} \sqrt{n_{l}+s}}{2} b_{n_{r} ; s+n_{l}-n_{r}-2}^{(2 k-2 l)}+\frac{\sqrt{n_{l}+s+1} \sqrt{n_{l}+s+2}}{2} b_{n_{r} ; s+n_{l}-n_{r}+2}^{(2 k-2 l)}\right) \text {, } \\
& H_{j j^{\prime}}^{(2 k+2)}=\sum_{l=0}^{k} \sum_{s=-2 k-1}^{2 k+1} b_{n_{l} ; s}^{(2 l)}\left(\left\{2 l(2 k-2 l)+\frac{\left(n_{l}+s\right)^{2}+n_{l}+s+1}{2}\right.\right. \\
& \left.+\frac{z_{c}^{2} \omega}{2}\left(2 n_{l}+2 s+1\right)\right\} b_{n_{r} ; s+n_{l}-n_{r}}^{(2 k-2 l)} \\
& +\frac{z_{c} \sqrt{\omega} \sqrt{n_{l}+s}}{\sqrt{2}}\left(-4 l+2 k-n_{l}-s\right) b_{n_{r} ; s+n_{l}-n_{r}-1}^{(2 k-2 l)} \\
& +\frac{z_{c} \sqrt{\omega} \sqrt{n_{l}+s+1}}{\sqrt{2}}\left(4 l-2 k-n_{l}-s-1\right) b_{n_{r} ; s+n_{l}-n_{r}+1}^{(2 k-2 l)} \\
& -\frac{\sqrt{n_{l}+s-1} \sqrt{n_{l}+s}}{2}\left(-4 l+2 k+z_{c}^{2} \omega\right) b_{n_{r} ; s+n_{l}-n_{r}-2}^{(2 k-2 l)} \\
& -\frac{\sqrt{n_{l}+s+1} \sqrt{n_{l}+s+2}}{2}\left(4 l-2 k+z_{c}^{2} \omega\right) b_{n_{r} ; s+n_{l}-n_{r}+2}^{(2 k-2 l)} \\
& +\frac{z_{c} \sqrt{\omega} \sqrt{n_{l}+s-2} \sqrt{n_{l}+s-1} \sqrt{n_{l}+s}}{\sqrt{2}} b_{n_{r} ; s+n_{l}-n_{r}-3}^{(2 k-2 l)} \\
& +\frac{z_{c} \sqrt{\omega} \sqrt{n_{l}+s+1} \sqrt{n_{l}+s+2} \sqrt{n_{l}+s+3}}{\sqrt{2}} b_{n_{r} ; s+n_{l}-n_{r}+3}^{(2 k-2 l)} \\
& -\frac{\sqrt{n_{l}+s-3} \sqrt{n_{l}+s-2} \sqrt{n_{l}+s-1} \sqrt{n_{l}+s}}{4} b_{n_{r} ; s+n_{l}-n_{r}-4}^{(2 k-2 l)} \\
& \left.-\frac{\sqrt{n_{l}+s+1} \sqrt{n_{l}+s+2} \sqrt{n_{l}+s+3} \sqrt{n_{l}+s+4}}{4} b_{n_{r} ; s+n_{l}-n_{r}+4}^{(2 k-2 l)}\right) .
\end{aligned}
$$

In step 5 we sequentially evaluate the solutions $b_{n ; s}^{(2 k)}$ and $\beta_{n}^{(2 k)}$ of the set of recurrence relations (22), (23) in each $k$ th order $\left(k=1, \ldots, k_{\max }\right)$ :

$$
\begin{aligned}
& f_{n ; 0}^{(2 k)}=0 \rightarrow \beta_{n}^{(2 k)} \\
& b_{n ; s \neq 0}^{(2 k)}=f_{n ; s}^{(2 k)} /(2 s) \\
& I_{i i}^{(2 k)}=\delta_{k 0} \rightarrow b_{n ; 0}^{(2 k)} .
\end{aligned}
$$


Table 4. The same as in Table 1 but for $H_{j j}(r)$

\begin{tabular}{lcccc}
$j, j$ & $H_{11}, 10^{-2}$ & $H_{22}, 10^{-2}$ & $H_{33}, 10^{-2}$ & $H_{44}, 10^{-1}$ \\
\hline$r^{-2} H_{j j}^{(2)}$ & 1.1562500 & 3.4687500 & 7.3437500 & 1.2781250 \\
$+r^{-4} H_{j j}^{(4)}$ & 1.1675830 & 3.5283837 & 7.5051513 & 1.3110092 \\
$+r^{-6} H_{j j}^{(6)}$ & 1.1677930 & 3.5299856 & 7.5110492 & 1.3125245 \\
$+r^{-8} H_{j j}^{(8)}$ & 1.1677976 & 3.5300324 & 7.5112738 & 1.3125967 \\
$+r^{-10} H_{j j}^{(10)}$ & 1.1677977 & 3.5300339 & 7.5112827 & 1.3126002 \\
\hline (n.v.) & 1.1677977 & 3.5300339 & 7.5112831 & 1.3126004 \\
\hline
\end{tabular}

In step 6, by substituting (12) with the coefficients $b_{n ; s}^{(2 k)}$ calculated at step $\mathbf{5}$ into the expressions for the matrix elements evaluated at step $\mathbf{4}$ and taking into account the above definition $r^{-2} E_{j}(r)=r^{-2}\left(\lambda_{j}(r)+c^{2}+f\right)=\omega \beta_{j}(r)$, i.e. $E_{j}^{(2 k)}=\omega \beta_{j}^{(2 k)}$, we produce the output containing the matrix elements as an expansion in inverse powers of $r$ for $k=0,1, \ldots, k_{\max }$ at $j, j^{\prime}=1, \ldots, j_{\max }$ :

$$
r^{-2} E_{j}(r)=\sum_{k=0}^{k_{\max }} \frac{E_{j}^{(2 k)}}{r^{2 k}}, H_{j j^{\prime}}(r)=\sum_{k=1}^{k_{\max }} \frac{H_{j j^{\prime}}^{(2 k)}}{r^{2 k}}, Q_{j j^{\prime}}(r)=\sum_{k=1}^{k_{\max }} \frac{Q_{j j^{\prime}}^{(2 k-1)}}{r^{2 k-1}} .
$$

The calculation described above was performed by the algorithm implemented in MAPLE up to $k_{\max }=8$. For example, the explicit expression of the desirable nonzero coefficients $E_{j}^{(2 k)}, H_{i j}^{(2 k)}=H_{j i}^{(2 k)}$ and $Q_{i j}^{(2 k-1)}=-Q_{j i}^{(2 k-1)}$ reads as $(j=n+1)$ :

$$
\begin{aligned}
& E_{j}^{(0)}=\omega(2 n+1), E_{j}^{(2)}=m^{2}-\frac{1}{4}-\frac{z_{c}^{2} \omega}{2}(2 n+1)-\frac{n^{2}+n+1}{2}, \\
& H_{j j}^{(2)}=\frac{z_{c}^{2} \omega}{2}(2 n+1)+\frac{n^{2}+n+1}{2}, \\
& H_{j j-1}^{(2)}=-\frac{z_{c} \sqrt{\omega} \sqrt{n} n}{\sqrt{2}}, \quad H_{j j-2}^{(2)}=-\frac{\omega z_{c}^{2} \sqrt{n-1} \sqrt{n}}{2} \\
& Q_{j j-1}^{(1)}=-\frac{z_{c} \sqrt{\omega} \sqrt{n}}{\sqrt{2}}, \quad Q_{j j-2}^{(1)}=\frac{\sqrt{n-1} \sqrt{n}}{2} .
\end{aligned}
$$

Tables 14 demonstrate the convergence of partial sums in the asymptotic expansions (27) of effective potentials $Q_{i j}(r)$ and $H_{i j}(r)$ calculated by the algorithm MATRA to the corresponding numerical values calculated by means of ODPEVP 9.

Remark 3. As follows from Eq. (28), the reduction of the problem (11) under the axial symmetry at fixed $m$ by means of the modified angular prolate spheroidal functions (4) at large $r$ leads to the asymptotic centrifugal term $\left(E_{j}^{(2)}+H_{j j}^{(2)}\right) r^{-2}=\left(m^{2}-1 / 4\right) r^{-2}$ in the effective potentials (9) of the set of radial equations (8). The latter term is characterized by the integer magnetic quantum number $m$. 


\section{Symbolic Algorithm for Evaluation the Asymptotic Forms of Radial Solutions}

In the procedure ASYMRS, using the above asymptotic expressions of the matrix elements, the asymptotic forms of the fundamental radial solutions $\chi_{j i_{o}}(r)$ of Eqs. (8) at small and large values of $r$ are calculated, and the needed boundary conditions for the reduced interval $\left[r_{\min }, r_{\max }\right]$ are generated. Here we consider the case of large $r$.

We find the asymptotic solution $\chi_{j i_{o}}^{a s}(r)$ at large $r \geq r_{\max }$ in the form

$$
\chi_{j i_{o}}^{a s}(r)=\left(\phi_{j i_{o}}(r)+\psi_{j i_{o}}(r) \frac{d}{d r}\right) R\left(p_{i_{o}}, r\right),
$$

where $p_{i_{o}}^{2}=2 E-\varepsilon_{i_{o}}^{t h}$ is the relative energy with respect to the threshold value $\varepsilon_{i_{o}}^{t h}=E_{i_{o}}^{(0)}$, and the function $R\left(p_{i_{o}}, r\right) \equiv R\left(p_{i_{o}}, r\right)$ satisfies the differential equation

$$
\frac{d^{2} R\left(p_{i_{o}}, r\right)}{d r^{2}}+\frac{2}{r} \frac{d R\left(p_{i_{o}}, r\right)}{d r}+\left(p_{i_{o}}^{2}+\frac{2 q}{r}-\frac{M_{2}}{r^{2}}\right) R\left(p_{i_{o}}, r\right)=0
$$

In (30) the asymptotic centrifugal term $M_{2} / r^{2}$ with the factor $M_{2}=m^{2}-1 / 4$ determines the order $\nu$ of the desirable solution

$$
R\left(p_{i_{o}}, r\right) \equiv R_{\nu}\left(p_{i_{o}}, r\right)=p_{i_{o}}^{-1 / 2} r^{-1}\left(\imath F_{\nu}\left(p_{i_{o}}, r\right)+G_{\nu}\left(p_{i_{o}}, r\right)\right) / 2,
$$

where $F_{\nu}\left(p_{i_{o}}, r\right)$ and $G_{\nu}\left(p_{i_{o}}, r\right)$ are the regular and irregular Coulomb functions of the half-integer order $\nu=m-1 / 2$ [11].

Remark 4. In the conventional 3D problem under spherical symmetry using the angular spherical harmonic functions leads to integer $M_{2}=l(l+1)$ and $\nu=l$ [12], whereas in the 3D problem under axial symmetry the angular oblate spheroidal functions at large $r$ lead to $M_{2}=0$ and $\nu=0$ [6. However, at small $r$ in both cases we have $M_{2}=l(l+1)$ and $\nu=l$.

In the case of $p_{i_{o}}=0$ and $q \neq 0$ the function $R\left(p_{i_{o}}, r\right)$ has the form

$$
R\left(p_{i_{o}}, r\right)=\pi^{1 / 2} r^{-1 / 2}\left(\imath J_{\nu^{\prime}}(\sqrt{8 q r})-Y_{\nu^{\prime}}(\sqrt{8 q r})\right) / 2,
$$

while in the case of $p_{i_{o}} \neq 0$ and $q=0$ it reads as

$$
R\left(p_{i_{o}}, r\right)=\pi^{1 / 2} 2^{-1 / 2} r^{-1 / 2}\left(\imath_{\nu^{\prime} / 2}\left(p_{i_{o}} r\right)-Y_{\nu^{\prime} / 2}\left(p_{i_{o}} r\right)\right) / 2 .
$$

Here $J_{\nu^{\prime}}$ and $Y_{\nu^{\prime}}$ are Bessel functions of the first and the second kind [10] of the order $\nu^{\prime}=\sqrt{1+4 M_{2}}, \nu^{\prime}=2 m$ at $M_{2}=m^{2}-1 / 4$ and $\nu^{\prime}=2 l+1$ at $M_{2}=l(l+1)$.

In step 1 substituting the function (29) into Eq. (8), using (30) and extracting the coefficients for the Coulomb function and its derivative, we arrive at two coupled differential equations with respect to the unknown functions $\phi_{j i_{o}}(r)$ and $\psi_{j i_{o}}(r)$. 
In step 2 we expand the functions $\phi_{j i_{o}}(r)$ and $\psi_{j i_{o}}(r)$ in inverse powers of $r$ :

$$
\phi_{j i_{o}}(r)=\sum_{k=0}^{k_{\max }} \phi_{j i_{o}}^{(k)} r^{-k}, \quad \psi_{j i_{o}}(r)=\sum_{k=0}^{k_{\max }} \psi_{j i_{o}}^{(k)} r^{-k} .
$$

After substituting the expansions (27), (31) into Eqs. (8) and equating the coefficients at the same powers of $r$, we compute a set of recurrence relations with respect to the unknown coefficients $\phi_{j i_{o}}^{(k)}$ and $\psi_{j i_{o}}^{(k)}$

$$
\left(p_{i_{o}}^{2}-2 E+E_{j}^{(0)}\right) \phi_{j i_{o}}^{(k)}=f_{j i_{o}}^{(k)}, \quad\left(p_{i_{o}}^{2}-2 E+E_{j}^{(0)}\right) \psi_{j i_{o}}^{(k)}=g_{j i_{o}}^{(k)},
$$

where the right-hand sides $f_{j i_{o}}^{(k)}$ and $g_{j i_{o}}^{(k)}$ are defined by the relations

$$
\begin{aligned}
& f_{j i_{o}}^{(k)}=2 p_{i_{o}}^{2}(k-1) \psi_{j i_{o}}^{(k-1)} \\
& \quad+\left((k-2)(k-3)+M_{2}-\left(E_{j}^{(2)}+H_{j j}^{(2)}\right)\right) \phi_{j i_{o}}^{(k-2)}+2 q(2 k-3) \psi_{j i_{o}}^{(k-2)} \\
& \quad-M_{2}(k-2) \psi_{j i_{o}}^{(k-3)}-\sum_{k^{\prime}=3}^{k}\left(E_{j}^{\left(k^{\prime}\right)}+H_{j j}^{\left(k^{\prime}\right)}\right) \phi_{j i_{o}}^{\left(k-k^{\prime}\right)} \\
& \quad+\sum_{j^{\prime}=1, j^{\prime} \neq j}^{j_{\max }} \sum_{k^{\prime}=1}^{k}\left[\left(\left(2 k-k^{\prime}-3\right) Q_{j j^{\prime}}^{\left(k^{\prime}-1\right)}-H_{j j^{\prime}}^{\left(k^{\prime}\right)}\right) \phi_{j^{\prime} i_{o}}^{\left(k-k^{\prime}\right)}\right. \\
& \left.\quad+\left(2 p_{i_{o}}^{2} Q_{j j^{\prime}}^{\left(k^{\prime}\right)}+4 q Q_{j j^{\prime}}^{\left(k^{\prime}-1\right)}\right) \psi_{j^{\prime} i_{o}}^{\left(k-k^{\prime}\right)}\right], \\
& \quad+g_{j i_{o}}^{(k)}=-2(k-1) \phi_{j i_{o}}^{(k-1)} \\
& \quad+M_{2} \phi_{j i_{o}}^{(k-2)}+\left(k(k-1)-\left(E_{j}^{(2)}+H_{j j}^{(2)}\right)\right) \psi_{j i_{o}}^{(k-2)} \\
& \quad-2 M_{2}(k-2) \psi_{j i_{o}}^{(k-3)}-\sum_{k^{\prime}=1}^{k}\left(E_{j}^{\left(k^{\prime}\right)}+H_{j j}^{\left(k^{\prime}\right)}\right) \psi_{j i_{o}}^{\left(k-k^{\prime}\right)} \\
& \quad \sum_{j^{\prime}=1, j^{\prime} \neq j}^{j_{\max }} \sum_{k^{\prime}=1}^{k}\left[\left(\left(2 k-k^{\prime}+1\right) Q_{j j^{\prime}}^{\left(k^{\prime}-1\right)}-H_{j j^{\prime}}^{\left(k^{\prime}\right)}\right) \psi_{j^{\prime} i_{o}}^{\left(k-k^{\prime}\right)}-2 Q_{j j^{\prime}}^{\left(k^{\prime}\right)} \phi_{j^{\prime} i_{o}}^{\left(k-k^{\prime}\right)}\right] .
\end{aligned}
$$

It should be noted that these relations differ from the case of $M_{2}=0$ [6] only by the terms containing $M_{2}=E_{i_{o}}^{(2)}+H_{i_{o} i_{0}}^{(2)}$.

In step 3 from equations (32) at $k=0$ we get the initial data for the recurrence procedure, including the special threshold case $2 E=E_{i_{o}}^{(0)}\left(p_{i_{o}}^{2}=0\right)$

$$
\phi_{j_{0} i_{o}}^{(0)}=\delta_{j_{0} i_{o}}, \quad \psi_{j_{0} i_{o}}^{(0)}=0, \quad p_{i_{o}}^{2}=2 E-E_{i_{o}}^{(0)} .
$$

The open channels have $p_{i_{o}}^{2} \geq 0$ whereas the close channels have $p_{i_{o}}^{2}<0$. Suppose that there are $N_{o} \leq j_{\max }$ open channels, i.e., $p_{i_{o}}^{2} \geq 0$ for $i_{o}=1, \ldots N_{o}$ and $p_{i_{o}}^{2}<0$ for $i_{o}=N_{o}+1, \ldots j_{\max }$. After substitution of (35) into (32) the recurrence relations for $k=1,2, \ldots, k_{\max }$ take the form

$$
\left(E_{j}^{(0)}-E_{i_{o}}^{(0)}\right) \phi_{j i_{o}}^{(k)}=f_{j i_{o}}^{(k)}, \quad\left(E_{j}^{(0)}-E_{i_{o}}^{(0)}\right) \psi_{j i_{o}}^{(k)}=g_{j i_{o}}^{(k)} .
$$


Step 4 performs the calculation of the coefficients $\phi_{j i_{o}}^{(k)}$ and $\psi_{j i_{o}}^{(k)}$ by a stepby-step procedure of solving equations (36) with r.h.s. determined by (33), (34), for $k=1,2, \ldots, k_{\max }$ :

$$
\begin{aligned}
& \phi_{j i_{o}}^{(k)}=\frac{f_{j i_{o}}^{(k)}}{E_{j}^{(0)}-E_{i_{o}}^{(0)}}, \quad \psi_{j i_{o}}^{(k)}=\frac{g_{j i_{o}}^{(k)}}{E_{j}^{(0)}-E_{i_{o}}^{(0)}}, \quad j \neq i_{o}, \\
& \left\{f_{i_{o} i_{o}}^{\left(k+1+\delta i_{i_{o}}\right)}=0, g_{i_{o} i_{o}}^{(k+1)}=0\right\} \rightarrow\left\{\phi_{i_{o} i_{o}}^{(k)}, \psi_{i_{o} i_{o}}^{(k)}\right\},
\end{aligned}
$$

where $\delta p_{i_{o}}=1$ at $p_{i_{o}}=0$ (threshold case) and $\delta p_{i_{o}}=0$ at $p_{i_{o}} \neq 0$.

The calculation was performed by means of the algorithm, implemented in MAPLE up to $k_{\max }=8$. Its output contains the elements at $k=0,1, \ldots, k_{\max }$. For example, for $k_{\max }=1$ we have the coefficients $\phi_{j i_{o}}^{(k)}$ and $\phi_{j i_{o}}^{(k)}$ in the form

$$
\begin{aligned}
& \phi_{j i_{o}}^{(1)}=0, \quad \psi_{j i_{o}}^{(1)}=\frac{2 Q_{j i_{o}}^{(1)}}{E_{i_{o}}^{(0)}-E_{j}^{(0)}} \\
& \phi_{i_{o} i_{o}}^{(1)}=0, \quad \psi_{i_{o} i_{o}}^{(1)}=-\left(1-\frac{\delta p_{i_{o}}}{3}\right) \sum_{j_{0}=\max \left(1, i_{o}-2\right), j_{0} \neq i_{o}}^{\min \left(j_{\max }, i_{o}+2\right)} Q_{i_{o} j_{0}}^{(1)} \psi_{j_{0} i_{o}}^{(1)}
\end{aligned}
$$

and substituting the asymptotic expressions (27) into the above equation, we arrive at the explicit expression of the desirable nonzero coefficients $\phi_{j i_{o}}^{(k)}$ and $\phi_{j i_{o}}^{(k)}\left(\right.$ for $\left.j_{\max } \geq i_{o}+2 k, i_{o}=n_{o}+1,\left\langle\hat{z} \mid i_{o}\right\rangle=\sqrt[4]{\omega} \Phi_{n_{o}}^{(0)}(\hat{z}), z^{\prime}=z-z_{c}=\frac{\hat{z}}{\sqrt{\omega}}-z_{c}\right)$ :

$$
\begin{aligned}
& \psi_{i_{o}-2 i_{o}}^{(1)}=-\frac{1}{2} \frac{\sqrt{n_{o}-1} \sqrt{n_{o}}}{2 \omega}=-\frac{1}{2}\left\langle i_{o}-2\left|\left(z-z_{c}\right)^{2}\right| i_{o}\right\rangle_{z}, \\
& \psi_{i_{o}-1 i_{o}}^{(1)}=\frac{z_{c} \sqrt{n_{o}}}{\sqrt{2} \sqrt{\omega}}=-\frac{1}{2}\left\langle i_{o}-1\left|\left(z-z_{c}\right)^{2}\right| i_{o}\right\rangle_{z}, \\
& \psi_{i_{o} i_{o}}^{(1)}=-\frac{1}{2}\left(1-\frac{\delta p_{i_{o}}}{3}\right)\left(\frac{2 n_{o}+1}{2 \omega}+z_{c}^{2}\right)=-\frac{1}{2}\left(1-\frac{\delta p_{i_{o}}}{3}\right)\left\langle i_{o}\left|\left(z-z_{c}\right)^{2}\right| i_{o}\right\rangle_{z}, \\
& \psi_{i_{o}+1 i_{o}}^{(1)}=\frac{z_{c} \sqrt{n_{o}+1}}{\sqrt{2} \sqrt{\omega}}=-\frac{1}{2}\left\langle i_{o}+1\left|\left(z-z_{c}\right)^{2}\right| i_{o}\right\rangle_{z}, \\
& \psi_{i_{o}+2 i_{o}}^{(1)}=-\frac{1}{2} \frac{\sqrt{n_{o}+1} \sqrt{n_{o}+2}}{2 \omega}=-\frac{1}{2}\left\langle i_{o}+2\left|\left(z-z_{c}\right)^{2}\right| i_{o}\right\rangle_{z} .
\end{aligned}
$$

Remark 5. The obtained results correspond to the asymptotic transformation of the arguments $r, \eta$ of the total function $\psi_{m i_{o}}^{a s}$ in terms of the asymptotic basis functions $\Phi_{j}^{a s}(\hat{z} ; r)$ from (12) at fixed magnetic quantum number $m$ :

$$
\begin{aligned}
\psi_{m i_{o}}^{a s}(r, \eta) & =\sum_{j=1}^{j_{\max }} \Phi_{j}^{a s}(\hat{z} ; r) \chi_{j i_{o}}^{a s}(r)=\sum_{j=1}^{j_{\max }} \Phi_{j}^{a s}(\hat{z} ; r)\left(\phi_{j i_{o}}(r)+\psi_{j i_{o}}(r) \frac{d}{d r}\right) R\left(p_{i_{o}}, r\right) \\
& =\sqrt[4]{\omega} \sqrt{r} \sum_{k=0}^{k_{\max }} r^{-k} \sum_{p=0}^{k} \sum_{j=1}^{j_{\max }} \Phi_{j}^{(p)}(\hat{z})\left(\phi_{j i_{o}}^{(k-p)}+\psi_{j i_{o}}^{(k-p)} \frac{d}{d r}\right) R\left(p_{i_{o}}, r\right) .
\end{aligned}
$$



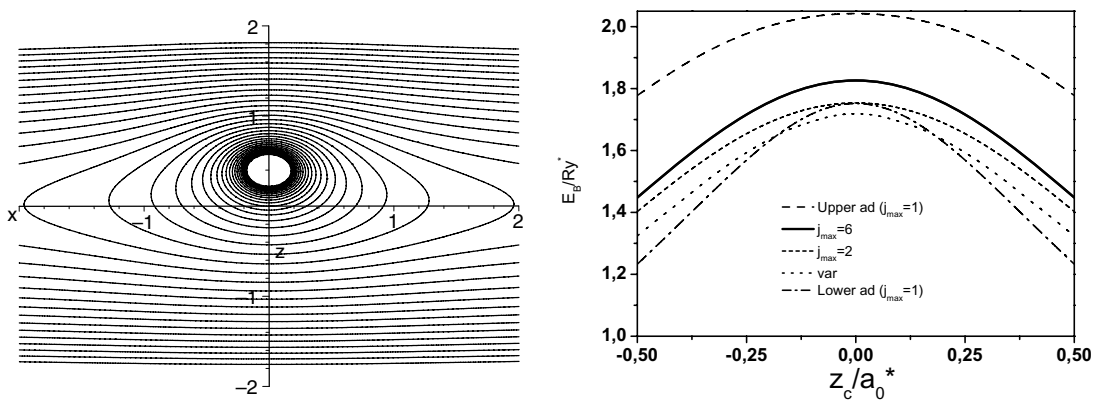

Fig. 1. Isolines of the potential energy surface as a function of two independent variables $\rho, z$ with shift of center of Coulomb potential along the variable $z$ on $z_{c}=0.4$ (left panel). The binding energy $-E_{B}=2 E-\varepsilon_{1}^{t h}\left(\mathrm{Ry}^{*}=5.2 \mathrm{meV}\right)$ versus position shift of Coulomb center of impurity by variable $z$ in interval $z_{c} \in[-0.5,0.5]\left(\mathrm{a}_{0}^{*}=102 \stackrel{\circ}{A}\right)$ at $q=1, \omega=3$ and $m=0$ (right panel). Dotted line is variational calculations [1, dash-dotted line is the adiabatic approximation $\left(j_{\max }=1\right)$, short-dashed line is the Kantorovich approximation (4) at $j_{\max }=2$ basis functions, solid line is the Kantorovich approximation (4) at $j_{\max }=6$ basis functions, dashed line is the crude adiabatic approximation $\left(j_{\max }=1\right.$, when the diagonal adiabatic positive correction $H_{11}(r)=0$ is neglected).

Taking into account the orthogonality (14) and completeness $\sum_{j}\left\langle\hat{z}^{\prime} \mid j\right\rangle\langle j \mid \hat{z}\rangle=$ $\delta\left(\hat{z}^{\prime}-\hat{z}\right)$ relations for the asymptotic basis functions $\Phi_{j}^{(0)}(\hat{z} ; r)$, we obtain the desirable asymptotic form of the total wave function at $p_{i_{o}} \hat{z} /(2 r) \ll 1$ and $k_{\max }=1$ :

$$
\begin{aligned}
& \psi_{m i_{o}}^{(a s)}(r, \eta)=\sqrt[4]{\omega} \sqrt{r} \sum_{j}\langle\hat{z} \mid j\rangle\left[\left\langle j \mid i_{o}\right\rangle-\frac{1}{2 r}\left\langle j\left|\left(z-z_{c}\right)^{2}\right| i_{o}\right\rangle \frac{d}{d r}\right] R\left(p_{i_{o}}, r\right) \\
& \approx \sqrt[4]{\omega} \sqrt{r} \Phi_{i_{o}}^{(0)}(\hat{z}) \chi_{i_{o} i_{o}}^{(a s)}\left(r-\frac{\left(z-z_{c}\right)^{2}}{2 r}\right) \\
& \approx \frac{\sqrt[4]{\omega}}{2\left(p_{i_{o}} \rho\right)^{1 / 2}} \Phi_{i_{o}}^{(0)}(\hat{z})\left(\imath F_{\nu}\left(p_{i_{o}}, \rho\right)+G_{\nu}\left(p_{i_{o}}, \rho\right)\right) .
\end{aligned}
$$

Thus, we obtain the needed compatibility conditions for the asymptotic solutions of scattering problems in the spherical coordinates $(r, \eta, \varphi)$ shifted by $z_{c}$ along $z$ axis and in the cylindrical coordinates $(\rho, z, \varphi)$

$$
\rho=r \sqrt{1-\left(z-z_{c}\right)^{2} / r^{2}}=r-\left(z-z_{c}\right)^{2} /(2 r)+O\left(r^{-2}\right),
$$

including the regular $F_{\nu}$ and irregular $G_{\nu}$ Coulomb functions of the half-integer order $\nu=m-1 / 2$ from Eq. (30).

It should be noted that at large $r$ the linearly independent functions (29) satisfy the Wronskian-type relation

$$
\mathbf{W}\left(\mathbf{Q}(r) ; \boldsymbol{\chi}^{*}(r), \boldsymbol{\chi}(r)\right)=\frac{\imath}{2} \mathbf{I}_{o o}
$$



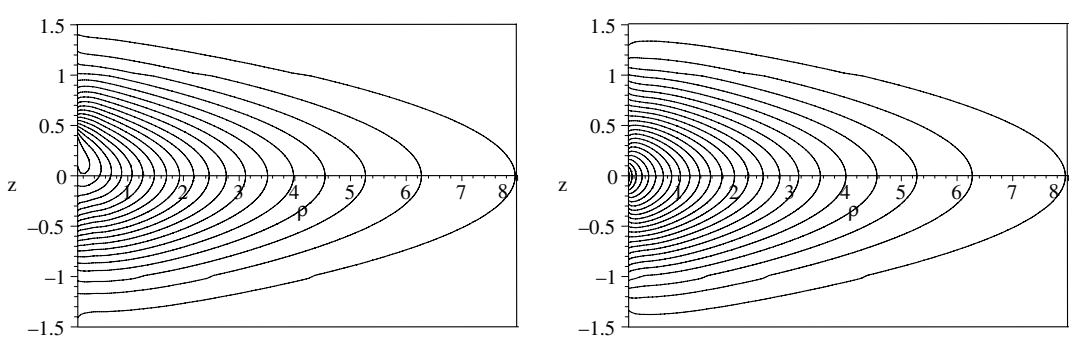

Fig. 2. Isolines of the ground-state wave function for the values of parameters $q=1$, $\omega=3$ and $m=0$. Left panel: $z_{c}=0.4$, Right panel: $z_{c}=0$.

where $\mathbf{W}\left(\bullet ; \boldsymbol{\chi}^{*}(r), \boldsymbol{\chi}(r)\right)$ is the generalized Wronskian with the long derivative defined as

$$
\mathbf{W}\left(\bullet ; \boldsymbol{\chi}^{*}(r), \boldsymbol{\chi}(r)\right)=r^{2}\left[\left(\boldsymbol{\chi}^{*}\right)^{T}\left(\frac{d \boldsymbol{\chi}}{d r}-\bullet \boldsymbol{\chi}\right)-\left(\frac{d \boldsymbol{\chi}^{*}}{d r}-\bullet \boldsymbol{\chi}^{*}\right)^{T} \chi\right] .
$$

These relations will be used to examine the desirable accuracy of the above expansion till $k_{\max }$ using KANTBP program implemented in FORTRAN [517] for numerical solving the boundary problem of discrete or continuous spectrum. The symbolic calculations of the above asymptotic expressions were performed using the codes MATRA and ASYMRS implemented in MAPLE, that generate FORTRAN codes of subroutines POTCAL and ASYMSC in KANTBP [517].

\section{Test Example}

The calculation for the GaAs parabolic quantum well was carried out with the values of parameters $q=1, \omega=3, m=0$, and $z_{c} \in[-0.5,0.5]$ in the reduced atomic units from [14] by applying the programs KANTBP [5] and ODPEVP [9]. These programs implementing the finite-element method to solve the boundaryvalue problems (8) -(11) and (5)-(7) were applied respectively on the grids $\Omega_{r}=$ $\{0(200) 1(200) 5(200) 100\}$ and $\Omega_{\eta}=\{-1(800) 1\}$ with the Lagrange elements of the order $p=4$ between the nodes. In the above grids $\Omega_{r}$ and $\Omega_{\eta}$, the number of grid elements is shown in the parentheses.

As follows from the theorem [13], for the ground state the adiabatic approximation $\left(j_{\max }=1\right)$ gives the upper bound for the energy, while in the so-called crude adiabatic approximation, when the diagonal adiabatic positive correction $H_{11}(r)=0$ is neglected, one gets the lower bound for the energy. The corresponding inverse estimators for the binding energy $-E_{B}=2 E-\varepsilon_{1}^{t h}$ (in units $R y^{*}=5.2 \mathrm{meV}$ ) in spherical coordinates are presented in Fig. 1. As one can see, these values are upper and lower estimates of the binding energy from the variational calculation [1]. The corresponding inverse lower estimators of the binding energy for increasing number of single-parameter basis functions $j_{\max }$ 
allow one to analyze the convergence rate of the method used for solving the boundary-value problem in the two-dimensional domain (see Fig. 2). In particular, the Kantorovich approximation (4) at $j_{\max }=10$ basis functions leads to the following inverse lower estimation of the binding energy $E_{B} / R y^{*}=1.82774$.

\section{Conclusion}

We presented the scheme for solving the boundary-value problem with discrete spectrum for a parabolic quantum well in the adiabatic representation. The upper and lower bounds for the energy of the ground state of the systems are obtained under the conditions of the shift of the Coulomb center in a given range of the parameter with respect to earlier variational estimates. It is shown that the rate of convergence depends significantly on the appropriate choice of the adiabatic basis parameterization taking the specific features of the considered problem into account. The presented results allow one to estimate the efficiency of the method and to prove the compatibility conditions (39) for asymptotic solutions of scattering problems in spherical and cylindrical coordinates. The software package developed is applicable to the investigation of semiconductor nanostructure models. Further development of the method and the software package is planned for solving the quasi-2D and quasi-1D boundary-value problems with both discrete and continuous spectrum, which are necessary for calculating the optical transition rates, channelling and transport characteristics in the models like quantum wells and quantum wires.

The authors thank Profs. V.L. Derbov, E.M. Kazaryan, A.A. Kostanyan and H.A. Sarkisyan for collaboration. The work was supported partially by RFBR (grants 07-01-00660 and 08-01-00604).

\section{References}

1. Kazaryan, E.M., Kostanyan, A.A., Sarkisyan, H.: Impurity optical absorption in parabolic quantim well. Physica E 28, 423-430 (2005)

2. Voss, H.: Numerical calculation of the electronic structure for three-dimensional quantum dots. Comput. Phys. Commun. 174, 441-446 (2006)

3. Wang, W., Hwang, T.-M., Jang, J.-C.: A second-order finite volume scheme for three dimensional truncated pyramidal quantum dot. Comput. Phys. Commun. 174, 371-385 (2006)

4. Gusev, A.A., Chuluunbaatar, O., Vinitsky, S.I., Derbov, V.L., Kazaryan, E.M., Kostanyan, A.A., Sarkisyan, H.A.: Adiabatic approach to the problem of a quantum well with a hydrogen - like impurity. Phys. Atomic Nuclei 72, 1600-1608 (2010)

5. Chuluunbaatar, O., Gusev, A.A., Abrashkevich, A.G., Amaya-Tapia, A., Kaschiev, M.S., Larsen, S.Y., Vinitsky, S.I.: KANTBP: A program for computing energy levels, reac-tion matrix and radial wave functions in the coupled-channel hyperspherical adia-batic approach. Comput. Phys. Commun. 177, 649-675 (2007)

6. Chuluunbaatar, O., Gusev, A.A., Gerdt, V.P., Rostovtsev, V.A., Vinitsky, S.I., Abrashkevich, A.G., Kaschiev, M.S., Serov, V.V.: POTHMF: A program for computing potential curves and matrix elements of the coupled adiabatic radial equations for a hydrogen-like atom in a homogeneous magnetic field. Comput. Phys. Commun. 178, 301-330 (2008) 
7. Chuluunbaatar, O., Gusev, A.A., Vinitsky, S.I., Abrashkevich, A.G.: KANTBP 2.0: New version of a program for computing energy levels, reaction matrix and radial wave functions in the coupled-channel hyperspherical adiabatic approach. Comput. Phys. Commun. 179, 685-693 (2008)

8. Gusev, A.I., Rempel, A.A.: Nanocrystalline materials. Cambridge Int. Sci, Cambridge (2004)

9. Chuluunbaatar, O., Gusev, A.A., Vinitsky, S.I., Abrashkevich, A.G.: ODPEVP: A program for computing eigenvalues and eigenfunctions and their first derivatives with respect to the parameter of the parametric self-adjoined Sturm-Liouville problem. Accepted in Comput. Phys. Commun. (2009), 10.1016/j.cpc.2009.04.017

10. Abramowitz, M., Stegun, I.A.: Handbook of Mathematical Functions. Dover, New York (1972)

11. Barnett, A.R.: KLEIN: Coulomb functions for real $\lambda$ and positive energy of high accuracy. Comput. Phys. Commun. 24, 141-159 (1981)

12. Barnett, A.R., Feng, D.H., Steed, J.W., Goldfarb, L.J.B.: Coulomb wave functions for all real $\eta$ and $\rho$. Comput. Phys. Comm. 8, 377-395 (1974)

13. Epstein, S.T.: Ground state energy of a molecule in adiabatic approximation. J. Chem. Phys. 144, 836-837 (1965) 